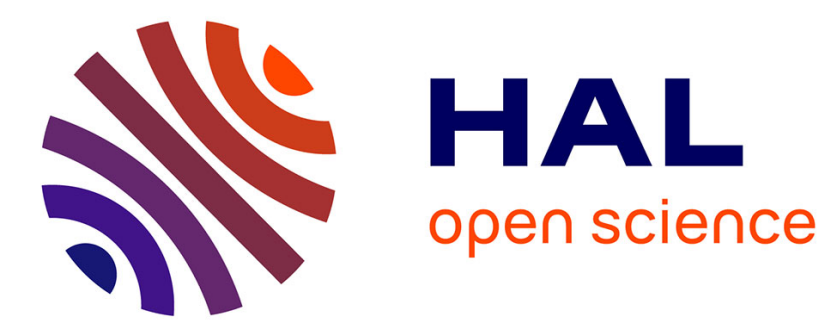

\title{
Level set discrete element method for three-dimensional computations with triaxial case study
}

Reid Kawamoto, Edward Andò, Gioacchino Cinno Viggiani, Jose Andrade

\section{To cite this version:}

Reid Kawamoto, Edward Andò, Gioacchino Cinno Viggiani, Jose Andrade. Level set discrete element method for three-dimensional computations with triaxial case study. Journal of the Mechanics and Physics of Solids, 2016, 91, pp.1-13. 10.1016/j.jmps.2016.02.021 . hal-02083354

\section{HAL Id: hal-02083354 https://hal.science/hal-02083354}

Submitted on 8 Apr 2019

HAL is a multi-disciplinary open access archive for the deposit and dissemination of scientific research documents, whether they are published or not. The documents may come from teaching and research institutions in France or abroad, or from public or private research centers.
L'archive ouverte pluridisciplinaire HAL, est destinée au dépôt et à la diffusion de documents scientifiques de niveau recherche, publiés ou non, émanant des établissements d'enseignement et de recherche français ou étrangers, des laboratoires publics ou privés. 


\title{
Level set discrete element method for three-dimensional computations with triaxial case study
}

\author{
Reid Kawamoto ${ }^{a}$, Edward Andò ${ }^{b}$, Gioacchino Viggiani ${ }^{b}$, José E. \\ Andrade ${ }^{a *}$ \\ ${ }^{a}$ Division of Engineering 8 Applied Science, California Institute of Technology, Pasadena, \\ CA 91125, USA \\ ${ }^{b}$ Grenoble-INP / UJF-Grenoble 1 / CNRS UMR 5521, Laboratoire 3SR, Grenoble, France
}

\begin{abstract}
In this paper, we outline the level set discrete element method (LS-DEM) which is a discrete element method variant able to simulate systems of particles with arbitrary shape using level set functions as a geometric basis. This unique formulation allows seamless interfacing with level set-based characterization methods as well as computational ease in contact calculations. We then apply LS-DEM to simulate two virtual triaxial specimens generated from XRCT images of experiments and demonstrate LS-DEM's ability to quantitatively capture and predict stress-strain and volume-strain behavior observed in the experiments.
\end{abstract}

\section{Introduction}

In this paper, we describe and validate the level set discrete element method (LS-DEM) which enables the simulation of systems of arbitrarily-shaped 3D particles using level set functions as a geometric basis. LS-DEM is similar to the classic discrete element method (DEM) [8] in that it simulates the kinematics and mechanics of a system of discrete particles, with the only difference being its ability to capture arbitrary shape as opposed to only spheres as in DEM. LS-DEM was motivated for three reasons:

1. Particle shape plays an enormous role in determining the macroscopic properties of an assembly, particularly strength, which has been shown both experimentally [5] and computationally [2], so having a method able to account for particle

\footnotetext{
*Corresponding author. E-mail: jandrade@caltech.edu (José E. Andrade)
} 


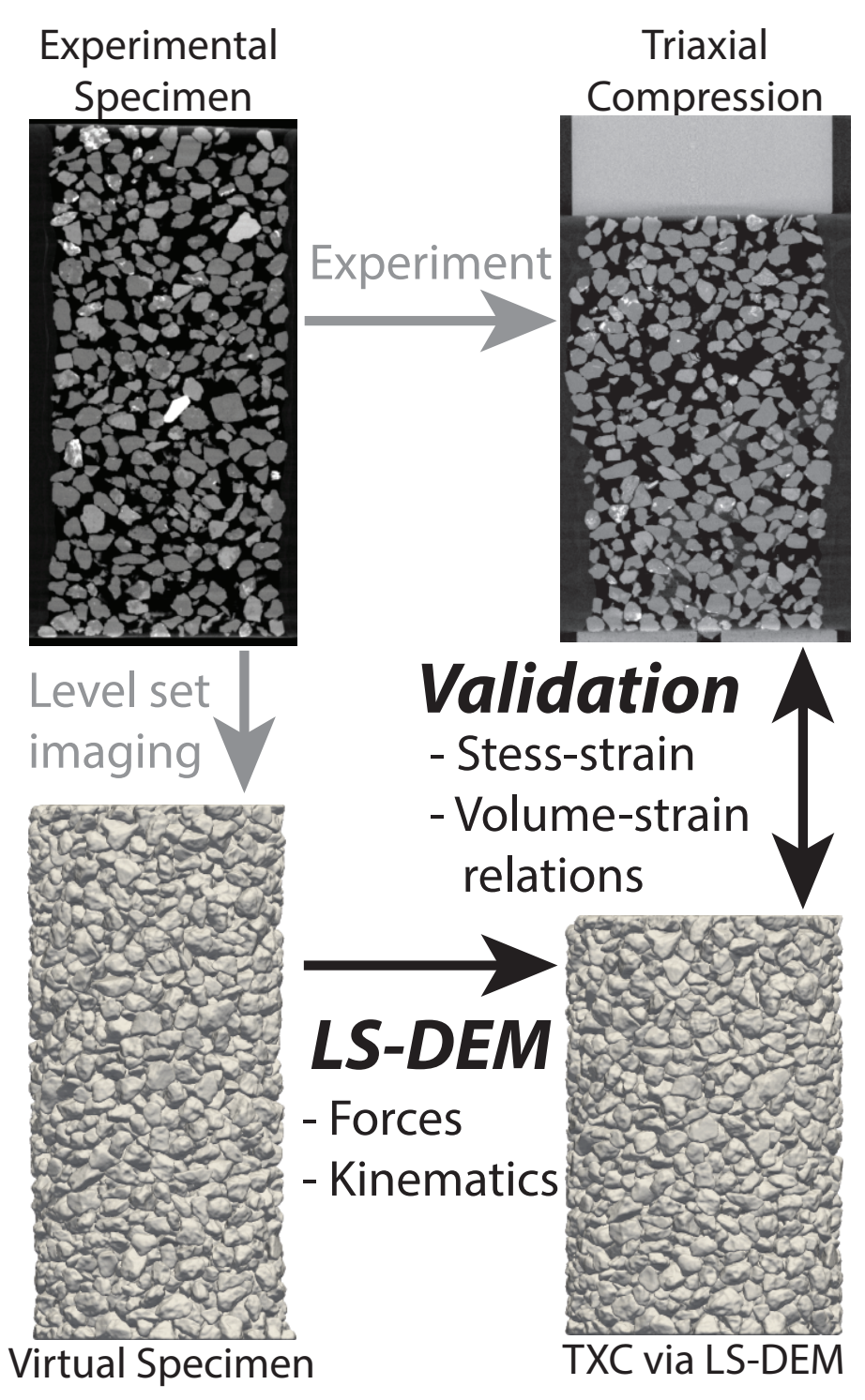

Figure 1: Illustration of this paper's contents, LS-DEM and its validation (bolditalicized), which are the links that allow us to fully connect the four areas between experiments and computations. Note that the experimental images are $2 \mathrm{D}$ slices of a $3 \mathrm{D}$ XRCT image, and the virtual specimen is $3 \mathrm{D}$. 
shape is paramount. Level set functions represent the next step in high-fidelity shape representation and are able to fully capture the complex morphology of real granular materials.

2. Recent developments in the characterization of grain assemblies from X-ray computed tomographic (XRCT) images of granular systems using level set imaging methods [21] have provided particular reason to develop a level set-based simulation method that can directly use the level set data from characterization of XRCT images as an input, avoiding the need to switch geometric formulations.

3. The level set framework is computationally efficient, even at high resolutions, due to its formulation.

With these motivations in mind, we describe the level set-based shape representation and contact algorithms unique to LS-DEM, then verify the method by applying it to two virtual assemblies obtained from XRCT images of experimental specimens. As shown in Figure 1, LS-DEM and its validation represent the the links in being able to fully bridge the gap between experiments and computations in discrete modeling with full-sized specimens.

\section{Level set discrete element method}

\subsection{Level set functions}

A level set function is a scalar-valued implicit function $\phi(\boldsymbol{p})$ whose value is the signed distance from a point $\boldsymbol{p}$ to an interface [15]. In the context of LS-DEM, the interface is the particle's surface. Consider a grain particle surface such as the one in Figure 2a. Contour lines can be added around the grain surface as in Figure 2b. These contour lines represent the distance or 'elevation' from the grain surface, positive outside the grain and negative inside the grain. Next, a grid can be superimposed over the contours as in Figure 2c and the elevation can be found at each grid point. Figure 2d illustrates the elevation at each grid point, and this is the level set function which is stored in computer memory and is the geometric basis of LS-DEM.

Although level set functions can be constructed through the method above, i.e., using point-distance formulas to arrive at Figure $2 \mathrm{~d}$, all of the level set functions in this paper were generated from XRCT images of experiments on real grains using level set-based imaging algorithms in [21].

Through interpolation of values at surrounding grid points, the value of the level set function at any point can be evaluated (Figure 2e). Define $\Omega^{+}=\{\boldsymbol{p} \mid \phi(\boldsymbol{p})>0\}$ the outside of the grain and $\Omega^{-}=\{\boldsymbol{p} \mid \phi(\boldsymbol{p})<0\}$ the inside of the grain. Then, the original grain surface (Figure $2 \mathrm{f}$ ) can be reconstructed by finding the set of points $\partial \Omega=\{\boldsymbol{p} \mid \phi(\boldsymbol{p})=0\}$ (the "zero level set") via interpolation. 


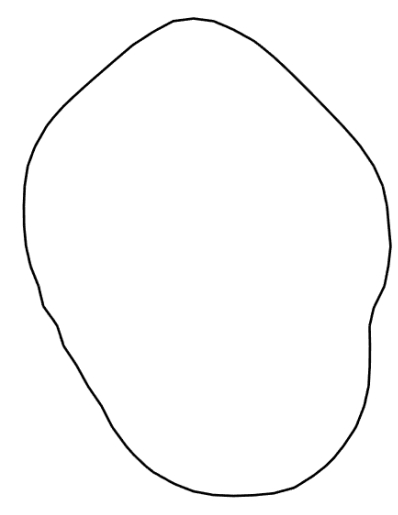

(a)

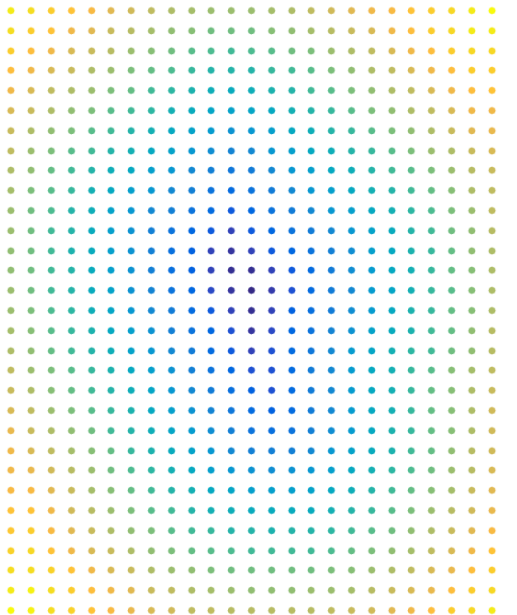

(d)

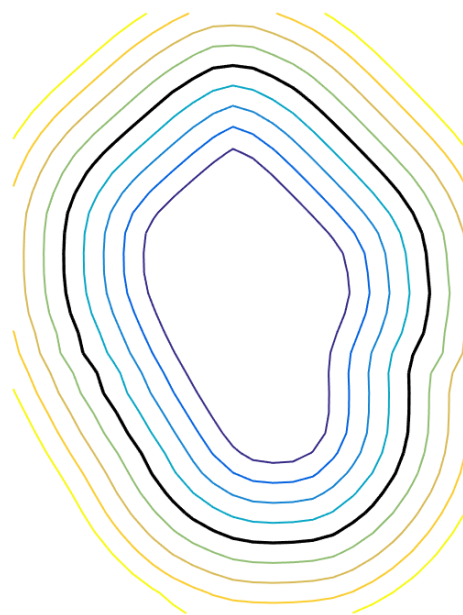

(b)

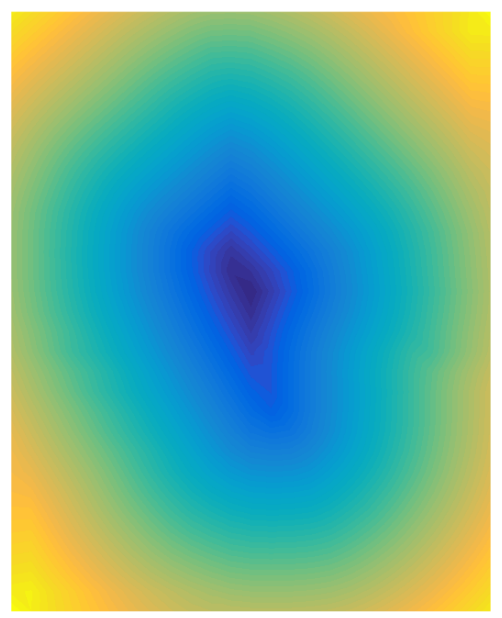

(e)

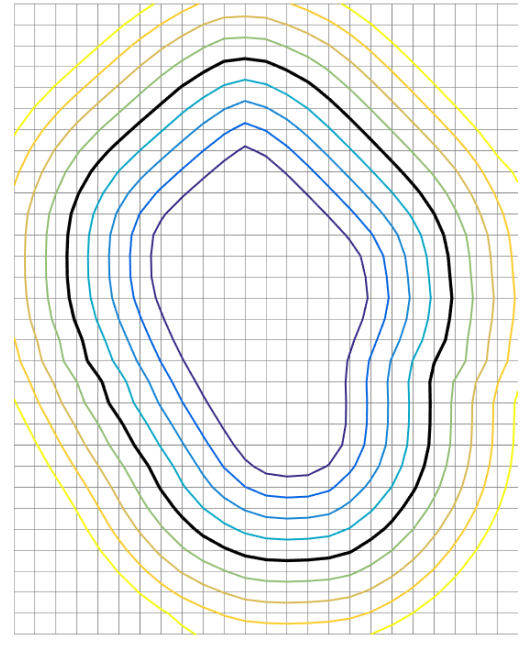

(c)

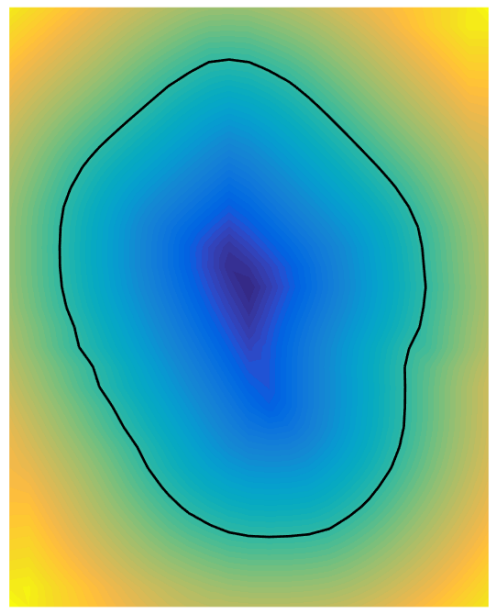

(f)

Figure 2: Illustration of a level set function. (a) Grain particle surface. (b) Contour lines representing signed distance from surface. (c) Superimposition on grid. (d) Discretized level set function. (e) Level set function with interpolation between grid points. (f) Reconstruction of original grain surface via interpolation. Note that the level set functions shown here are 2D for illustrative purposes only.

\subsection{Interpolation in level set functions}

For use in the level set discrete element method, we must be able to compute two quantities from a level set function $\phi$ : its value $\phi(\boldsymbol{p})$ and its gradient $\boldsymbol{\nabla} \phi(\boldsymbol{p})$ at any point $\boldsymbol{p}$ within its grid boundaries. This is done through interpolation of values of the discretized level set function at grid points surrounding $\boldsymbol{p}$. Any order of interpolation can be used, but linear interpolation was used here for its simplicity and speed. Let:

1. $\phi$ be stored on a uniform grid with grid spacing $g$ in all directions. 


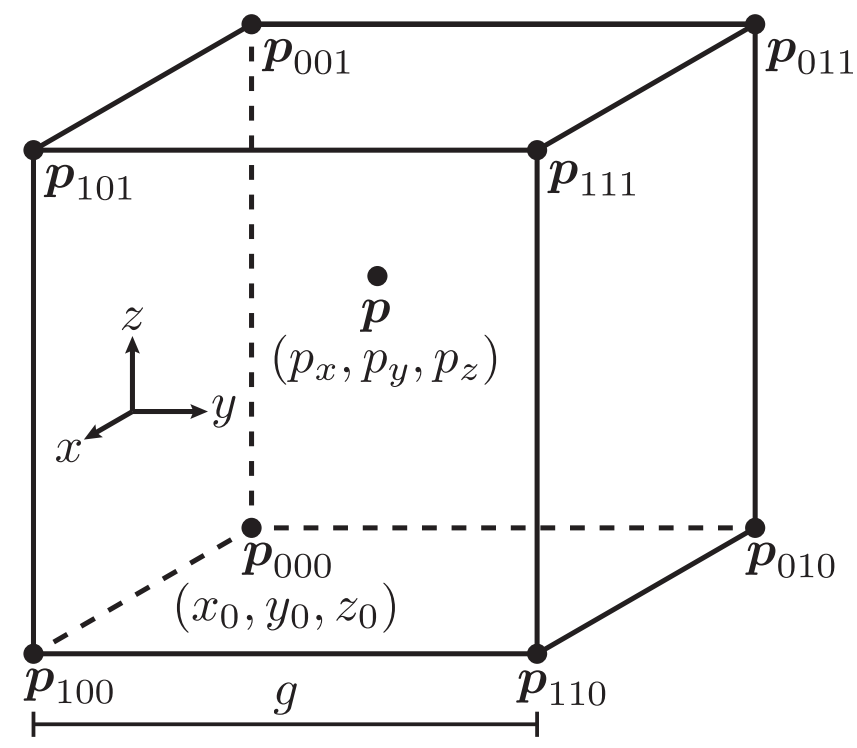

Figure 3: Schematic of point $\boldsymbol{p}$ with surrounding grid points $\boldsymbol{p}_{a b c}$.

2. $\boldsymbol{p}$ be a point in space with components $p_{x}, p_{y}$, and $p_{z}$ and surrounded by grid points $\boldsymbol{p}_{a b c}$ with $a, b, c \in\{0,1\}$ as shown in Figure 3 . Furthermore, let $\boldsymbol{p}_{000}$ have components $\left(x_{0}, y_{0}, z_{0}\right)$.

3. $x=\frac{\left(p_{x}-x_{0}\right)}{g}, y=\frac{\left(p_{y}-y_{0}\right)}{g}, z=\frac{\left(p_{z}-z_{0}\right)}{g}$

4. $\phi_{a b c}=\phi\left(\boldsymbol{p}_{a b c}\right)$ for convenience in notation.

Using trilinear interpolation to find $\phi(\boldsymbol{p})$,

$$
\phi(\boldsymbol{p})=\sum_{a=0}^{1} \sum_{b=0}^{1} \sum_{c=0}^{1} \phi_{a b c}[(1-a)(1-x)+a x][(1-b)(1-y)+b y][(1-c)(1-z)+c z]
$$

The gradient of the level set function $\boldsymbol{\nabla} \phi(\boldsymbol{p})$, using trilinear interpolation, is

$$
\boldsymbol{\nabla} \phi(\boldsymbol{p})=\left(\begin{array}{c}
\sum_{a=0}^{1} \sum_{b=0}^{1} \sum_{c=0}^{1} \phi_{a b c}(2 a-1)[(1-b)(1-y)+b y][(1-c)(1-z)+c z] \\
\sum_{a=0}^{1} \sum_{b=0}^{1} \sum_{c=0}^{1} \phi_{a b c}[(1-a)(1-x)+a x](2 b-1)[(1-c)(1-z)+c z] \\
\sum_{a=0}^{1} \sum_{b=0}^{1} \sum_{c=0}^{1} \phi_{a b c}[(1-a)(1-x)+a x][(1-b)(1-y)+b y](2 c-1)
\end{array}\right)
$$

Note that the interpolation functions to find $\phi(\boldsymbol{p})$ and $\boldsymbol{\nabla} \phi(\boldsymbol{p})$ are not functions of grid size. Therefore, the time complexity of these calculations are constant; they do not increase if $\phi$ is refined to a finer grid. 


\subsection{Inertial properties}

The inertial properties, i.e., mass, center of mass, and moment of inertia, of a given grain must be known for its use in LS-DEM. These quantities are computed directly from the grain's level set function. Define the smoothed Heaviside function $H(\phi)$ as

$$
H(\phi)=\left\{\begin{array}{cc}
0 & \text { if } \phi<-\epsilon \\
\frac{1}{2}\left(1+\frac{\phi}{\epsilon}+\frac{\sin \left(\frac{\pi \phi}{\epsilon}\right)}{\pi}\right) & \text { if }-\epsilon<\phi<\epsilon \\
1 & \text { if } \phi>\epsilon
\end{array}\right.
$$

where $\epsilon$ is a smoothness parameter. $\epsilon=1.5$ was used in this paper. The mass of a grain of uniform density $\rho$ and grid spacing $g$ represented by level set function $\phi$ is

$$
m=\rho g^{3} \sum_{i=1}^{I} \sum_{j=1}^{J} \sum_{k=1}^{K} H\left(\phi\left(x_{i}, y_{j}, z_{k}\right)\right)
$$

where $\phi\left(x_{i}, y_{j}, z_{k}\right)$ is the value of $\phi$ at grid point $\left(x_{i}, y_{j}, z_{k}\right)$, and $I, J$, and $K$ are the number of grid points in the $x, y$, and $z$ directions, respectively, of $\phi$. In other words, the mass is proportional to the summation of $H(\phi)$ over every grid point of $\phi$. The components of its center of mass are

$$
\begin{aligned}
& c_{x}=\frac{\rho g^{3}}{m} \sum_{i=1}^{I} \sum_{j=1}^{J} \sum_{k=1}^{K} H\left(\phi\left(x_{i}, y_{j}, z_{k}\right)\right) x_{i} \\
& c_{y}=\frac{\rho g^{3}}{m} \sum_{i=1}^{I} \sum_{j=1}^{J} \sum_{k=1}^{K} H\left(\phi\left(x_{i}, y_{j}, z_{k}\right)\right) y_{j} \\
& c_{z}=\frac{\rho g^{3}}{m} \sum_{i=1}^{I} \sum_{j=1}^{J} \sum_{k=1}^{K} H\left(\phi\left(x_{i}, y_{j}, z_{k}\right)\right) z_{k}
\end{aligned}
$$

Finally, the components of its moment of inertia are 


$$
\begin{aligned}
I_{11} & =\rho g^{3} \sum_{i=1}^{I} \sum_{j=1}^{J} \sum_{k=1}^{K} H\left(\phi\left(x_{i}, y_{j}, z_{k}\right)\right)\left[\left(y_{j}-c_{y}\right)^{2}+\left(z_{k}-c_{z}\right)^{2}\right] \\
I_{22} & =\rho g^{3} \sum_{i=1}^{I} \sum_{j=1}^{J} \sum_{k=1}^{K} H\left(\phi\left(x_{i}, y_{j}, z_{k}\right)\right)\left[\left(x_{i}-c_{x}\right)^{2}+\left(z_{k}-c_{z}\right)^{2}\right] \\
I_{33} & =\rho g^{3} \sum_{i=1}^{I} \sum_{j=1}^{J} \sum_{k=1}^{K} H\left(\phi\left(x_{i}, y_{j}, z_{k}\right)\right)\left[\left(x_{i}-c_{x}\right)^{2}+\left(y_{j}-c_{y}\right)^{2}\right] \\
I_{23}=I_{32} & =-\rho g^{3} \sum_{i=1}^{I} \sum_{j=1}^{J} \sum_{k=1}^{K} H\left(\phi\left(x_{i}, y_{j}, z_{k}\right)\right)\left(y_{j}-c_{y}\right)\left(z_{k}-c_{z}\right) \\
I_{13}=I_{31} & =-\rho g^{3} \sum_{i=1}^{I} \sum_{j=1}^{J} \sum_{k=1}^{K} H\left(\phi\left(x_{i}, y_{j}, z_{k}\right)\right)\left(x_{i}-c_{x}\right)\left(z_{k}-c_{z}\right) \\
I_{12}=I_{21} & =-\rho g^{3} \sum_{i=1}^{I} \sum_{j=1}^{J} \sum_{k=1}^{K} H\left(\phi\left(x_{i}, y_{j}, z_{k}\right)\right)\left(x_{i}-c_{x}\right)\left(y_{j}-c_{y}\right)
\end{aligned}
$$

\subsection{Boundary node discretization}

LS-DEM uses a node-to-surface contact algorithm that is utilized in finite element models [12] as well as discrete element models [2] for the handling of nonconvex particles with multiple contact points as well as computational ease, whereby nodes are seeded onto the surface $\partial \Omega$ of each particle (Figure 4 ). The density of nodes on a given particle is a matter of choice and has implications on particle behavior; however, we find that seeding with a maximum node-to-node spacing of less than $d / 10$, where $d$ is the particle diameter, is adequate to capture particle morphology as higher nodal densities have a negligible impact on behavior. Contact is then determined by checking each node of a master particle against the boundary of a slave particle for penetration. Because each node is checked for contact, the computational cost of contact is proportional to of the number of nodes seeded onto the master particle.

Note that the number of nodes seeded onto a particle does not change its underlying geometry, which is defined by its level set function, unlike polyhedra and clumping methods where changing the number of vertices or spheres completely modifies their geometries. Thus, more advanced schemes such as adaptive seeding near areas of contact during time integration are possible if such precision is desired.

\subsection{Contact}

As mentioned in the previous section, contact in LS-DEM is handled through a nodeto-surface contact algorithm. Let grain $i$ have nodes $\boldsymbol{m}_{a}^{i}$ with $\{a \in \mathbb{Z} \mid 1 \leq a \leq A\}$, 


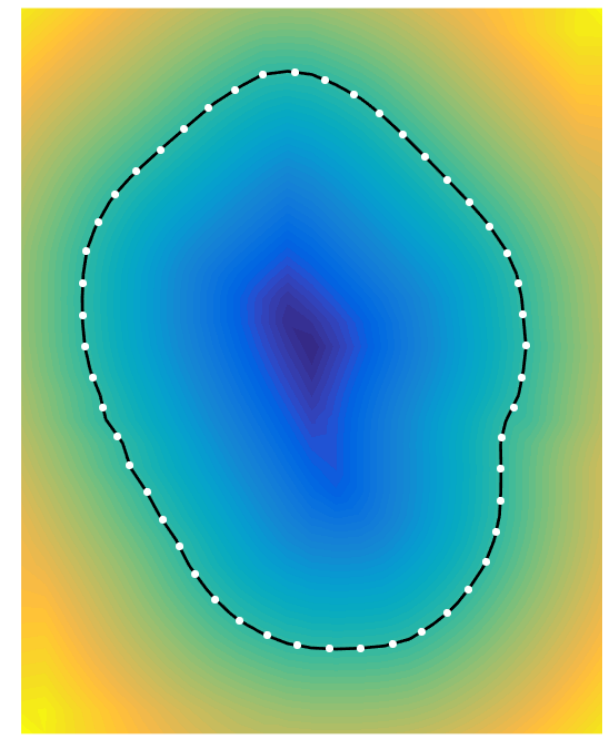

Figure 4: Example of boundary node discretization with nodes in white seeded on the grain surface, shown in 2D for illustrative purposes only.

where $A$ is the number of nodes seeded onto $i$. Contact is determined between master grain $i$ and slave grain $j$ by checking all nodes $\boldsymbol{m}_{a}^{i}$ of grain $i$ with the level set function $\phi^{j}$ of grain $j$. Then,

$$
\begin{aligned}
d_{a}^{j, i} & =\phi^{j}\left(\boldsymbol{m}_{a}^{i}\right) \\
\hat{\boldsymbol{n}}_{a}^{j, i} & =\frac{\boldsymbol{\nabla} \phi^{j}\left(\boldsymbol{m}_{a}^{i}\right)}{\left\|\boldsymbol{\nabla} \phi^{j}\left(\boldsymbol{m}_{a}^{i}\right)\right\|}
\end{aligned}
$$

where $d_{n}^{j, i}$ and $\hat{\boldsymbol{n}}_{a}^{j, i}$ are the penetration distance and outward contact normal of $j$, respectively, between grains $i$ and $j$ at node $\boldsymbol{m}_{a}^{i}$ (see Figure 5). These contact equations are very simple and easy to compute due to the formulation of the level set function, whose value at any point represents the distance from that point to the surface, and its gradient at any point represents, in principle, the unit outward normal at that point. However, due to the level set function's discrete nature, the magnitude of $\boldsymbol{\nabla} \phi^{j}\left(\boldsymbol{m}_{a}^{i}\right)$ is very close, but not equal, to unity and therefore is normalized.

If at least one node $\boldsymbol{m}_{a}^{i}$ of master grain $i$ is penetrating slave grain $j$, that is, if $\exists \boldsymbol{m}_{a}^{i} \mid \phi^{j}\left(\boldsymbol{m}_{a}^{i}\right)<0$, then we consider the two grains to be in contact, and thus, interparticle forces must be computed.

\subsection{Forces and moments}

To compute forces from penetrations, any contact model can be used, but we used a linear elastic contact model for the purposes of this study. Thus, the contact normal 


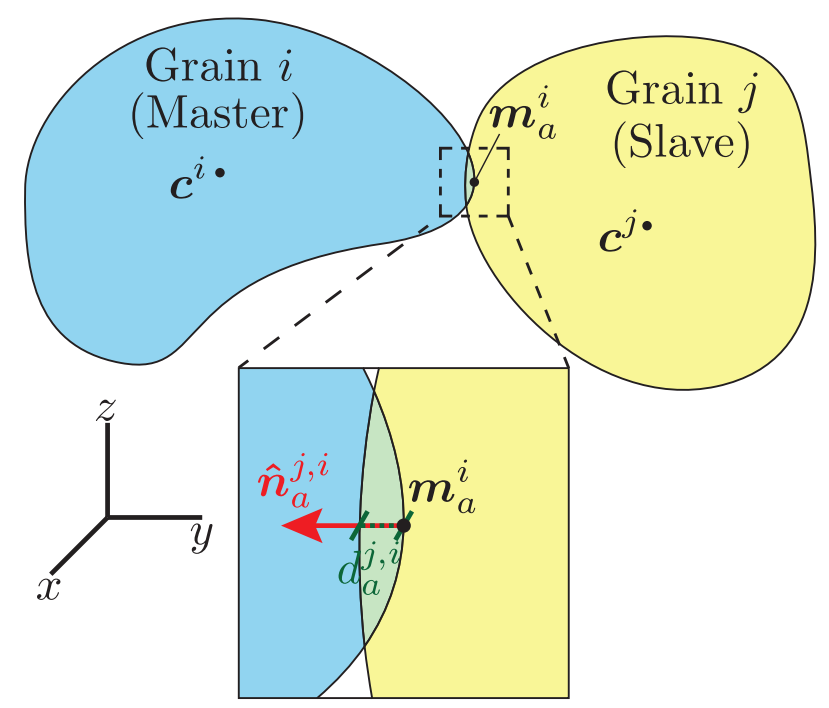

Figure 5: Illustration of two contacting grains.

force contribution from node $\boldsymbol{m}_{a}^{i}$ on grain $i$ is

$$
\boldsymbol{F}_{n, a}^{i}=\left\{\begin{array}{cc}
-k_{n} d_{a}^{j, i} \hat{\boldsymbol{n}}_{a}^{j, i} & \text { if } d_{a}^{j, i}<0 \\
0 & \text { else }
\end{array}\right.
$$

where $k_{n}$ is the normal contact stiffness. By action and reaction, the contribution of contact normal force $\boldsymbol{F}_{n, a}^{j}$ from node $\boldsymbol{m}_{a}^{i}$ on grain $j$ is

$$
\boldsymbol{F}_{n, a}^{j}=-\boldsymbol{F}_{n, a}^{i}
$$

The moment $\boldsymbol{M}_{n, a}^{i}$ contributed by the contact normal force $\boldsymbol{M}_{n, a}^{i}$ at node $\boldsymbol{m}_{a}^{i}$ on grain $i$ is

$$
\boldsymbol{M}_{n, a}^{i}=\left(\boldsymbol{m}_{a}^{i}-\boldsymbol{c}^{i}\right) \times \boldsymbol{F}_{n, a}^{i}
$$

where $\boldsymbol{c}^{i}$ is the centroid of grain $i$. Similarly, the moment $\boldsymbol{M}_{n, a}^{j}$ contributed by the contact normal force at node $\boldsymbol{m}_{a}^{i}$ on grain $j$ is

$$
\boldsymbol{M}_{n, a}^{j}=\left(\boldsymbol{m}_{a}^{i}-\boldsymbol{c}^{j}\right) \times \boldsymbol{F}_{n, a}^{j}
$$

where $\boldsymbol{c}^{j}$ is the centroid of grain $j$. For the calculation of shear and frictional forces, LS-DEM uses a Coulomb friction model similar to $[2,8]$. The relative velocity $\boldsymbol{v}_{a}$ of node $\boldsymbol{m}_{a}^{i}$ to grain $j$ is

$$
\boldsymbol{v}_{a}=\boldsymbol{v}^{i}+\boldsymbol{\omega}^{i} \times\left(\boldsymbol{m}_{a}^{i}-\boldsymbol{c}^{i}\right)-\boldsymbol{v}^{j}-\boldsymbol{\omega}^{j} \times\left(\boldsymbol{m}_{a}^{i}-\boldsymbol{c}^{j}\right)
$$

where $\boldsymbol{v}^{i}, \boldsymbol{v}^{j}, \boldsymbol{\omega}^{i}$, and $\boldsymbol{\omega}^{j}$ are the translational and angular velocities of grains $i$ and $j$. The incremental shear displacement $\Delta \boldsymbol{s}_{a}$ is then

$$
\Delta \boldsymbol{s}_{a}=\left[\boldsymbol{v}_{a}-\left(\boldsymbol{v}_{a} \cdot \hat{\boldsymbol{n}}_{a}^{j, i}\right) \hat{\boldsymbol{n}}_{a}^{j, i}\right] \Delta t
$$


The shear force $\boldsymbol{F}_{s, a}^{i}$ on grain $i$ contributed by node $\boldsymbol{m}_{a}^{i}$ is updated as such:

$$
\boldsymbol{F}_{s, a}^{i} \leftarrow \boldsymbol{Z} \boldsymbol{F}_{s, a}^{i}-k_{s} \Delta \boldsymbol{s}_{a}
$$

where $\boldsymbol{Z}$ is the rotation matrix that rotates the normal vector $\hat{\boldsymbol{n}}_{a}^{j, i}$ at the current timestep to the normal vector at the previous timestep and $k_{s}$ is the shear contact stiffness. The Coulomb friction law dictates $\boldsymbol{F}_{s, a}^{i}$ be capped at a fraction of the normal force $\boldsymbol{F}_{n, a}^{i}$ :

$$
\boldsymbol{F}_{s, a}^{i} \leftarrow \frac{\boldsymbol{F}_{s, a}^{i}}{\left\|\boldsymbol{F}_{s, a}^{i}\right\|} \min \left(\left\|\boldsymbol{F}_{s, a}^{i}\right\|, \mu\left\|\boldsymbol{F}_{n, a}^{i}\right\|\right)
$$

where $\mu$ is the interparticle friction coefficient. By action and reaction,

$$
\boldsymbol{F}_{s, a}^{j}=-\boldsymbol{F}_{s, a}^{i}
$$

The moment $\boldsymbol{M}_{s, a}^{i}$ contributed by node $\boldsymbol{m}_{a}^{i}$ 's shear force on grain $i$ is

$$
\boldsymbol{M}_{s, a}^{i}=\left(\boldsymbol{m}_{a}^{i}-\boldsymbol{c}^{i}\right) \times \boldsymbol{F}_{s, a}^{i}
$$

Similarly, the the moment $\boldsymbol{M}_{s, a}^{j}$ contributed by node $\boldsymbol{m}_{a}^{i}$, shear force on grain $j$ is

$$
\boldsymbol{M}_{s, a}^{j}=\left(\boldsymbol{m}_{a}^{i}-\boldsymbol{c}^{j}\right) \times \boldsymbol{F}_{s, a}^{j}
$$

The total contact force on grain $i$ is found by summing all nodal contact forces:

$$
\boldsymbol{F}_{t o t}^{i}=\sum_{a=1}^{A}\left(\boldsymbol{F}_{n, a}^{i}+\boldsymbol{F}_{s, a}^{i}\right)
$$

By action and reaction,

$$
\boldsymbol{F}_{t o t}^{j}=-\boldsymbol{F}_{t o t}^{i}
$$

The total contact moment on each grain is found by summing all nodal contact moments:

$$
\begin{aligned}
& \boldsymbol{M}_{\text {tot }}^{i}=\sum_{a=1}^{A}\left(\boldsymbol{M}_{n, a}^{i}+\boldsymbol{M}_{s, a}^{i}\right) \\
& \boldsymbol{M}_{t o t}^{j}=\sum_{a=1}^{A}\left(\boldsymbol{M}_{n, a}^{j}+M_{s, a}^{j}\right)
\end{aligned}
$$




\subsection{Motion}

Given a grain's inertial properties and the force and moment on it, the translational velocity, angular velocity, position, and rotation of the grain are updated using an appropriate time integration scheme. In this paper, the scheme described in [2, 22] was used to update the positions of the center of mass and nodes of each grain, so it is not included here for the sake of brevity.

It is important to note that, to minimize computational cost, the level set function of each grain is never updated as it moves; each level set function remains in a reference configuration. To accommodate this, when computing contact, the nodes $\boldsymbol{m}_{a}^{i}$ of grain $i$ (in the global frame) are moved temporarily into the reference configuration of grain $j$ 's level set function. From there, contact forces and moments are found (in the reference configuration of grain $j$ ) and then moved back to the global frame.

\section{Case study: Triaxial compression test}

We tested the validity of LS-DEM by simulating a triaxial compression (TXC) test using XRCT data taken from two real TXC experiments on a Martian-like sand. Our goal was to capture, through LS-DEM, both the axial stress-axial strain and volumetric strain-axial strain relations measured in the experiment. We calibrated the parameters of the LS-DEM model to replicate the results of one experiment, then used those parameters to predict the behavior of the other experiment.

\subsection{The experiment}

Two cylindrical specimens, each $11 \mathrm{~mm}$ in diameter and $24 \mathrm{~mm}$ in height, of a Martianlike sand were compressed isotropically to $100 \mathrm{kPa}$, then triaxially compressed at an axial strain rate of $\dot{\epsilon}_{1}=0.1 \% / \mathrm{s}$ and a constant radial pressure of $\sigma_{3}=100 \mathrm{kPa}$. 3DXRCT images with voxel edge length $31.1 \mu \mathrm{m}$ were taken of each specimen at the onset of axial strain. The stress-strain and volume-strain relations are plotted in Figure 6. The difference in behavior of the two specimens is attributed to the difference in the number of grains and therefore initial porosity of the two specimens; the looser specimen had 2,773 grains and an initial porosity of $41 \%$, while the denser specimen had 3,158 grains and an initial porosity of $36 \%$. 


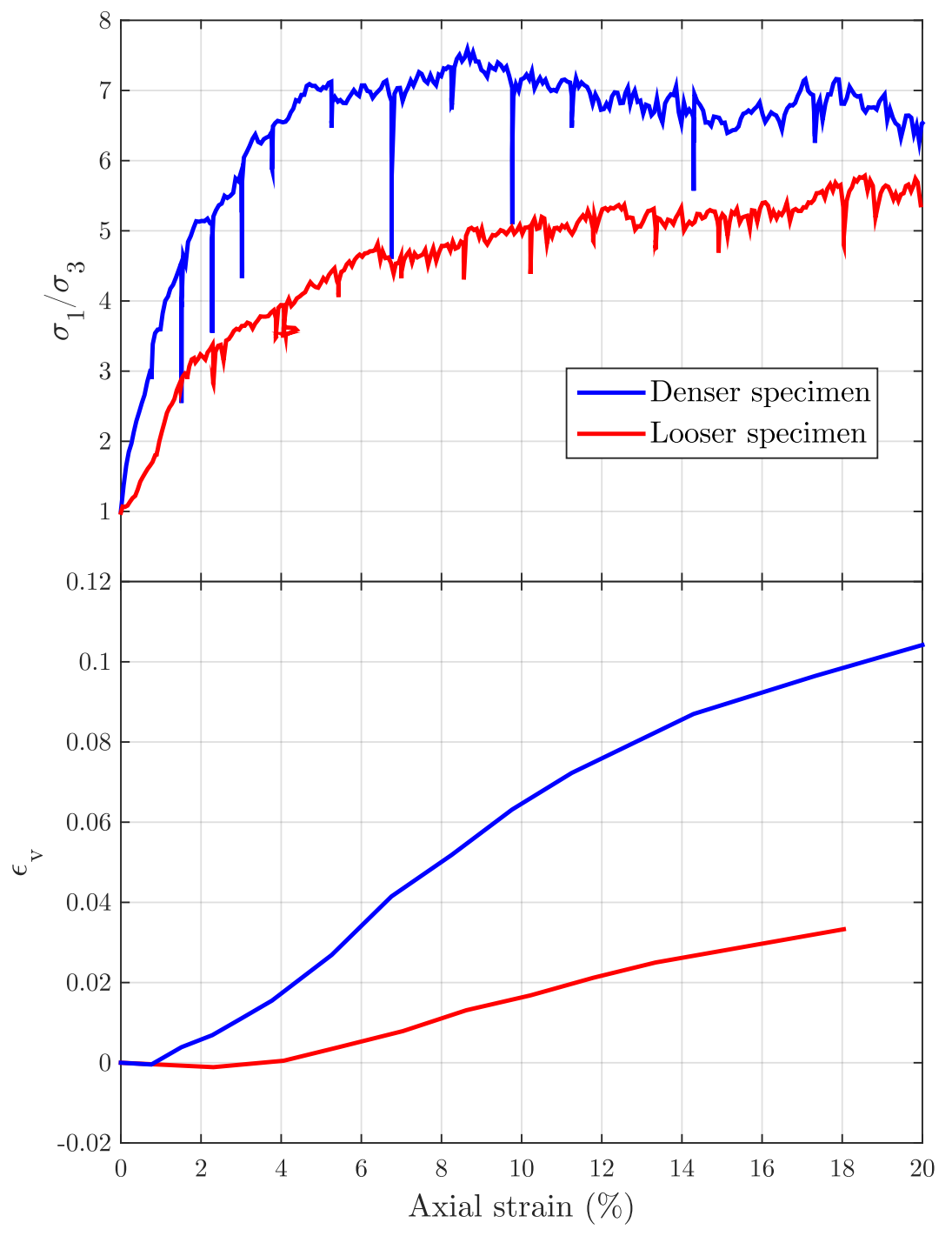

Figure 6: Stress-strain and volume-strain results of experiments. Note: experimental volume-strain data for the looser specimen was stopped at $18 \%$.

\subsection{LS-DEM calibration of looser specimen}

We applied the characterization technique described in [21] to produce level set functions of every grain from the XRCT image of the looser specimen at the onset of triaxial compression; in other words, we generated a virtual specimen where each grain was represented 1:1 in both shape and position as in Figure 7. Our goal was to calibrate the values of the interparticle normal stiffness $k_{n}$ and interparticle friction $\mu$ in an LSDEM simulation to match experimental results. Grain density was set at $2500 \mathrm{~kg} / \mathrm{m}^{3}$ and shear stiffness $k_{s}$ was held at $0.9 k_{n}$. While we were not able to reproduce the $\dot{\epsilon}_{1}=0.1 \% / s$ strain rate of the experiment due to computational limitations, as such a low strain rate would take prohibitively long to simulate, values of $\dot{\epsilon}_{1}$, global damp- 
ing, and $\Delta t$ were chosen to maintain quasi-static conditions, numerical stability, and computational tractability [20]. Rigid, frictionless walls were used and wall stiffness was set to be the same as grain stiffness $k_{n}$. We applied isotropic compression to the virtual specimen to a pressure of $100 \mathrm{kPa}$, then axially compressed the assembly to $\epsilon_{1}=20 \%$ while maintaining radial pressure $\sigma_{3}=100 \mathrm{kPa}$, the final configuration of which is shown in Figure 7. The stress-strain and volume-strain relations are plotted in Figure 8 with three different values each of $k_{n}$ and $\mu$.
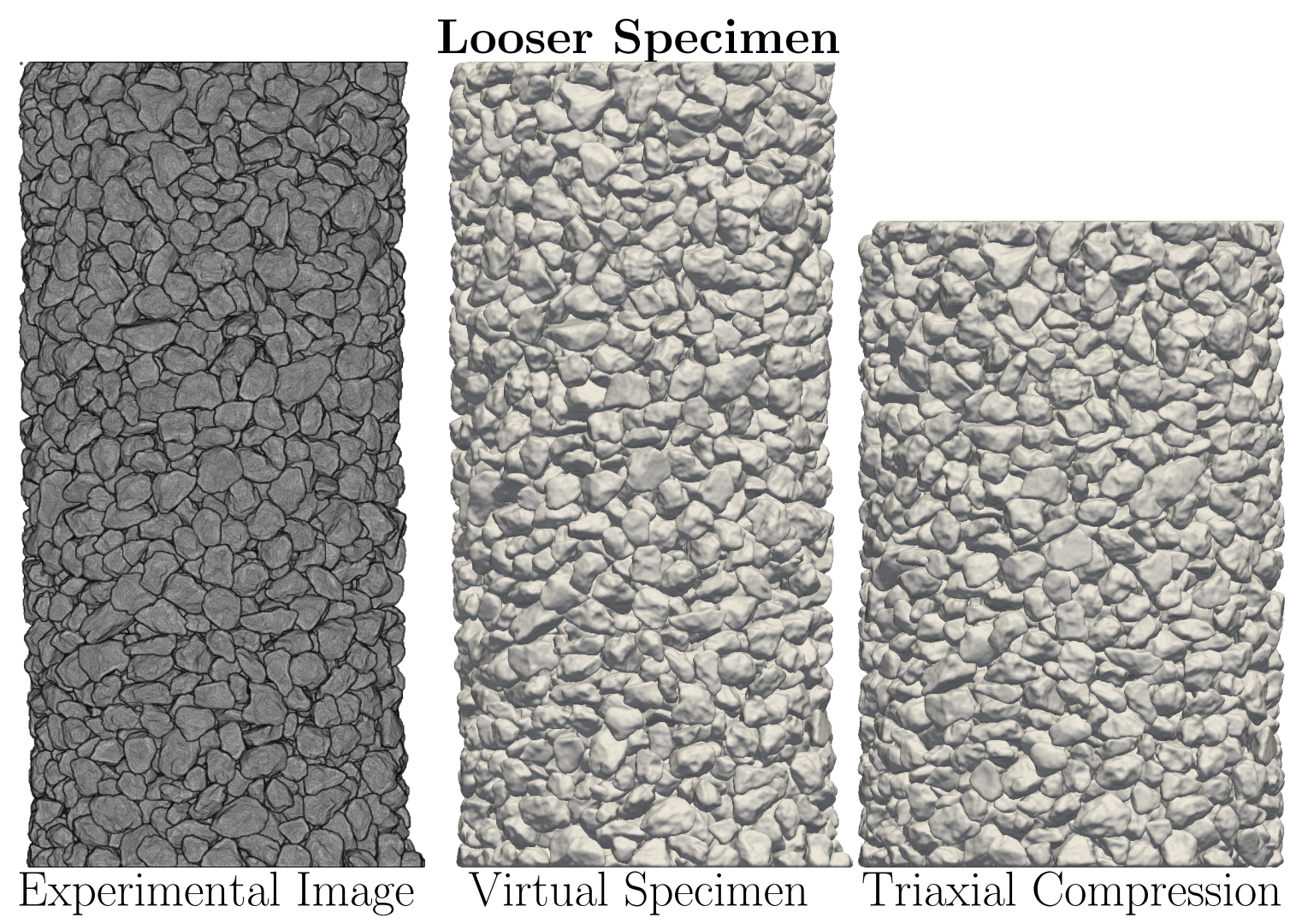

Figure 7: Left: 3D rendering of XRCT image of uncompressed looser specimen. Center: Virtual level set function representation of looser specimen. Right: Virtual specimen after triaxial compression. 

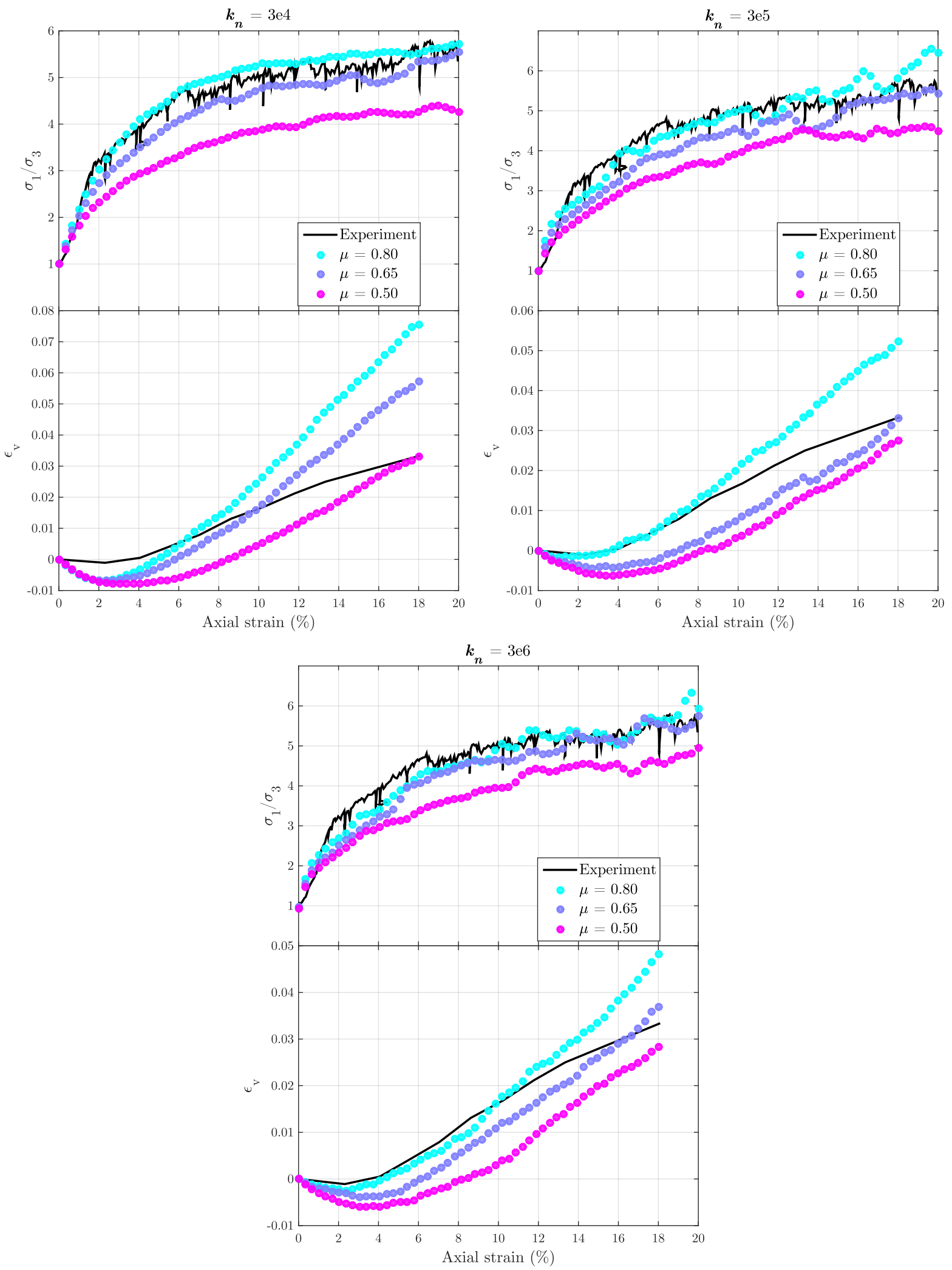

Figure 8: Stress-strain and volume-strain relations for LS-DEM simulations on looser specimen. 
From Figure 8, the values of $k_{n}$ and $\mu$ that resulted in the closest match in both stress-strain and volume-strain behavior were $3 \times 10^{6} \mathrm{~N} / \mathrm{m}$ and 0.65 , respectively. The contact stiffness $k_{n}$ overall did not have a particularly large effect of the behavior of the specimen, and a value of $3 \times 10^{6} \mathrm{~N} / \mathrm{m}$ seems reasonable especially in the context of DEM. As for the the interparticle friction coefficient $\mu$, while there is a wealth of experimental data on macroscopic rock friction at high pressures [3, 10], there is not much research at the low pressures and small length scales in the regime of this case study. Grain-scale experiments performed on quartz, a relatively "smooth" particle, have found an interparticle friction coefficient of about 0.24 [18]. While an interparticle coefficient of friction of 0.65 seems quite high in that context, the sand used in this case study's experiment consisted of unweathered rock fragments meant to mimic those of Mars. It is therefore not unreasonable that the grains would have a high amount of surface roughness unable to be captured by the resolution of the XRCT image, which manifests itself in a high coefficient of interparticle friction, especially at low pressures where friction is highly dependent on surface roughness [3].

\subsection{LS-DEM simulation of denser specimen}

We then sought to predict the experimental results of the denser specimen by using the calibrated values obtained from simulations on the looser specimen to simulate the denser specimen. The characterization process was repeated: from its XRCT image, we generated a virtual specimen of the denser specimen as shown in Figure 9. We then repeated the LS-DEM process of isotropic compression and triaxial compression with the calibrated values of $k_{n}=3 \times 10^{6}, \mu=0.65$ and all other parameters the same as before. The stress-strain and volume strain relations are plotted in Figure 10.

As Figure 10 indicates, using the calibrated parameters gives a reasonable prediction of the behavior of the denser specimen (right), but it is not as accurate as the results from the looser specimen with which we used to calibrate (left).

\section{Discussion}

In terms of assessing the validity of our LS-DEM model, further investigation can be done to determine if indeed the calibrated parameters $k_{n}$ and $\mu$ are reasonable by performing experiments on individual grains of the specimens using apparatuses and procedures in $[7,18]$ to compute $k_{n}$ and $\mu$, respectively. Also, while slight bulging was seen in the experimental specimens, in our simulations, we used rigid, straight walls, which prevented bulging from happening computationally. It would be interesting to see if the implementation of a flexible membrane instead of rigid walls in our simulations could improve results.

The shape-based nature of LS-DEM makes it rife with possibilities in studying the mechanical properties of granular assemblies. One area that looks promising is grain breakage and communition, experiments of which at the grain-scale have been 


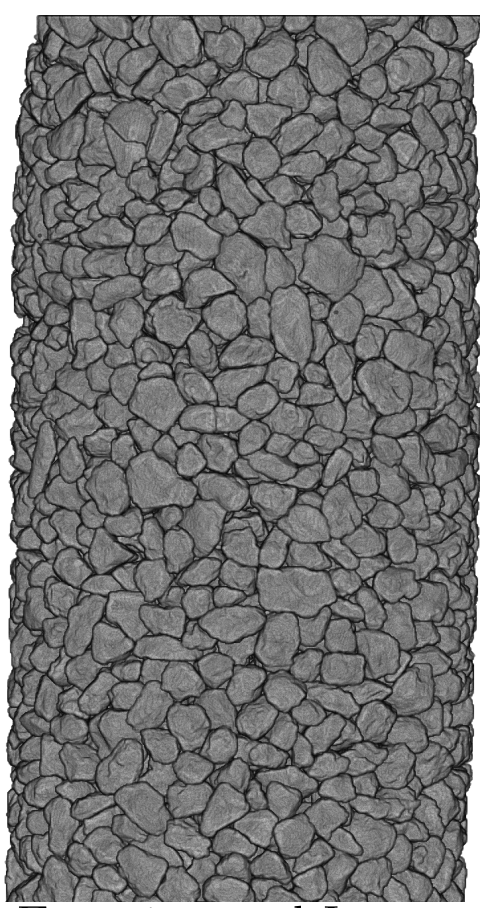

Experimental Image

\section{Denser Specimen}

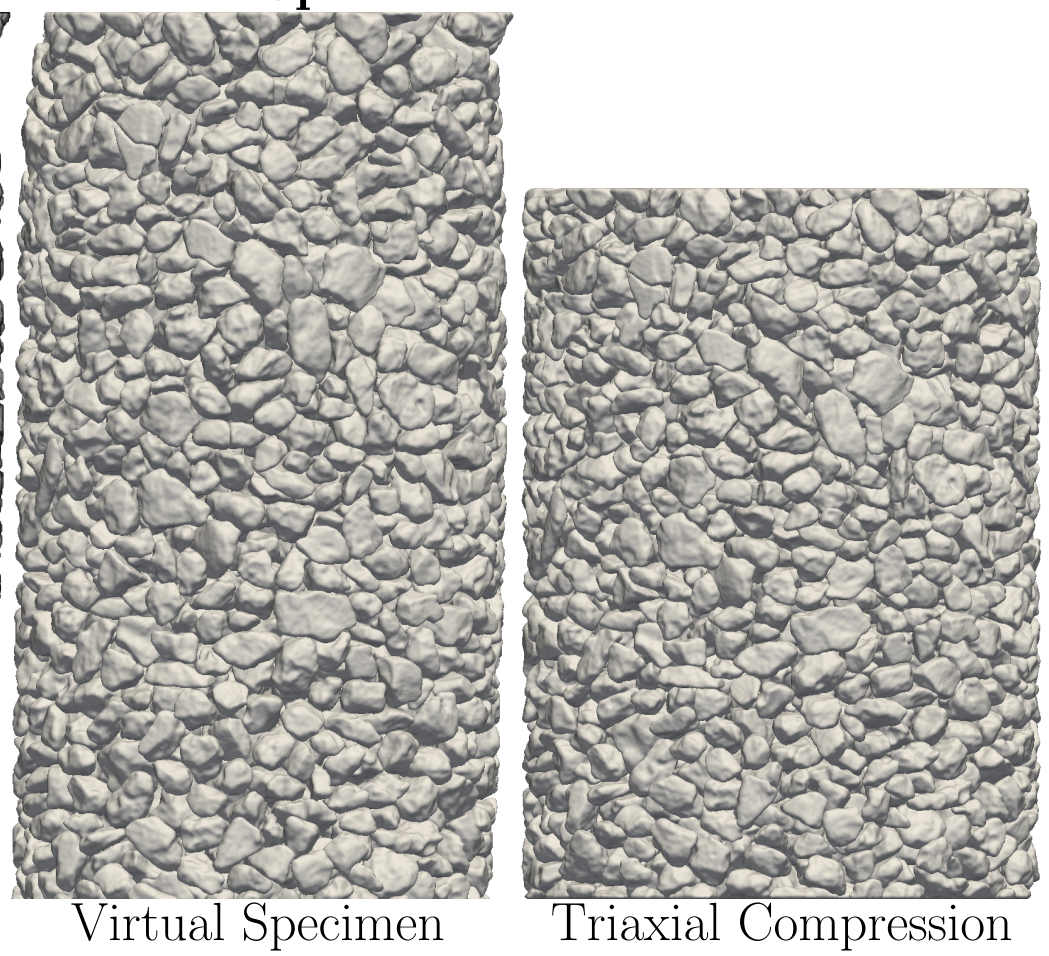

Figure 9: Left: 3D rendering of XRCT image of uncompressed denser specimen. Center: Virtual level set function representation of denser specimen. Right: Virtual specimen after triaxial compression.

performed $[6,7,16]$, some of which were also investigated using DEM. Determining fracture criteria (a function of contact forces [11], coordination number [4, 17], and probability [23, 24]) and direction of fracture planes (a function of Mohr-Coulomb failure surfaces [19] and location of contact forces) for grains of arbitrary morphology is not a trivial task. However, once fracture criteria and fracture planes have been determined, the level set framework is convenient for modeling grain breakage as fracture planes can be represented by level set functions which then can be used to split a grain using binary operations between the level set function of the grain and the level set functions of the fracture planes, which would allow replications of exact fracture patterns that occur in experiments. Another area in which LS-DEM could be applied is in multiscale methods, such as the one developed and implemented in [1, 13], or using LS-DEM to infer continuum quantities such as dilatancy and macroscopic friction angle to shed light on how these continuum properties originate from the grain scale. Essentially, the potential applications of LS-DEM fall under the same umbrella as those of classic DEM, but with its ability to capture shape, LS-DEM will hopefully enable us to arrive at a deeper, more quantitative understanding of the behavior of granular materials. 


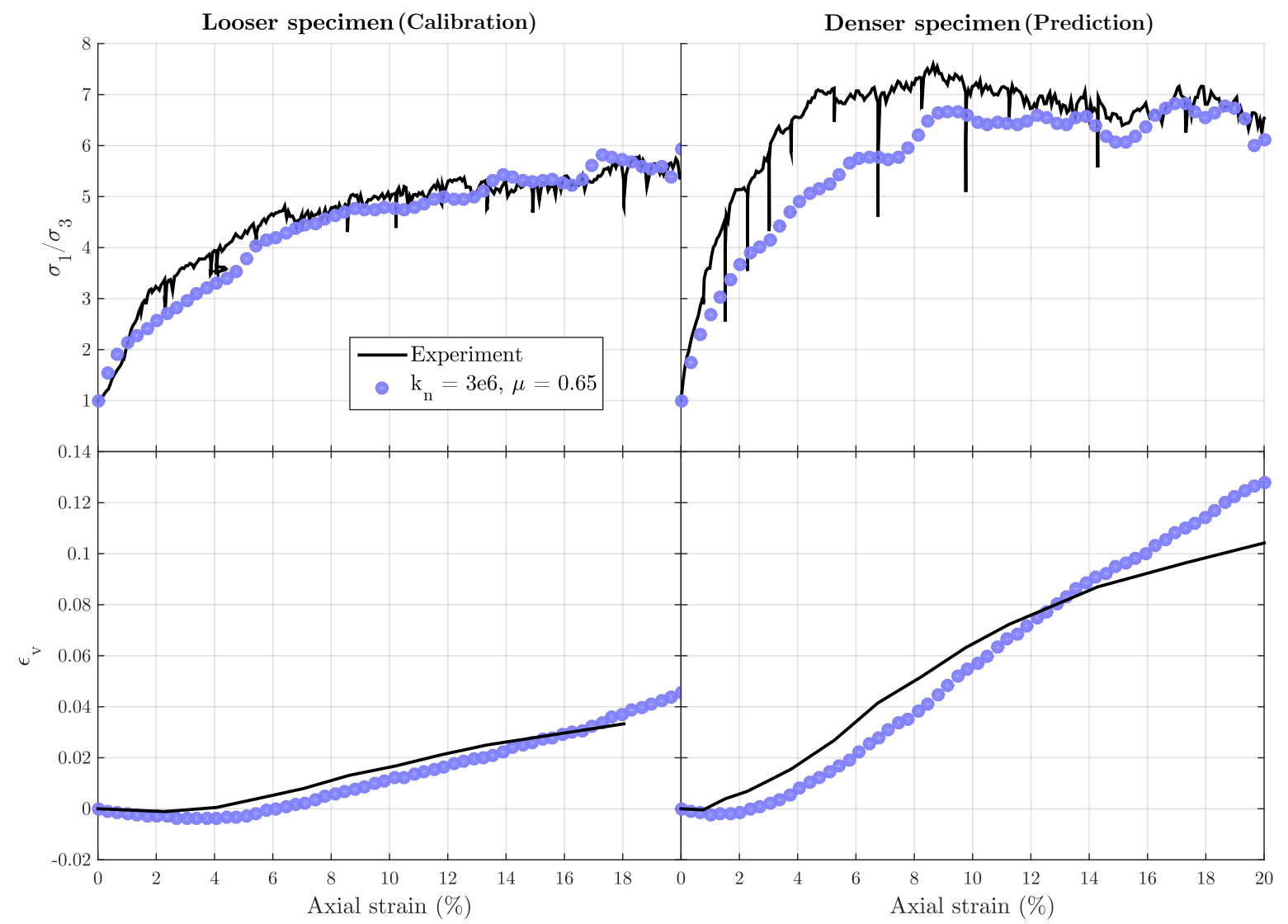

Figure 10: Stress-strain and volume-strain relations for LS-DEM simulations on looser (left) and denser (right) specimens with $k_{n}=3 \times 10^{6}$ and $\mu=0.65$.

There are also possible extensions and studies of LS-DEM in the computational realm. The computational time complexity of LS-DEM does not scale with increased grid resolution is and relatively low compared to that of polyhedral or NURBS-based methods as contact detection does not require traversal a tree of bounding volumes $[9,14]$ but rather is constant and only requires a lookup of values at grid points. In this sense, level set functions work essentially as lookup tables of penetration distances and contact normals where values between grid points are estimates found via interpolation. Two interesting questions arise as a result of this. One, how does grid fineness relate to accuracy? In this paper, grid fineness was held constant as the grains' level set functions resided on grids at the same resolution as the XRCT images themselves. Two, what effect does memory consumption have on the computational cost and limitations of LS-DEM? Because LS-DEM requires an underlying grid with a value of $\phi$ at every grid point, it consumes a large amount of memory. A $40 \times 40 \times 40$ level set function requires 64,000 values to be stored, while a NURBS curve with 20 knots in each direction or a polyhedra with 400 vertices requires only 1,200 values to be stored, which is a memory consumption of less than $2 \%$ than that of the aforementioned level set 
function's memory footprint. Even though LS-DEM's contact algorithm has constant time complexity with respect to grid resolution, a large memory footprint nonetheless may lead to increased computation time due to cache misses. It could even lead to crashes if memory overflows, limiting the amount of grains able to be simulated. However, many clusters today have large amounts of memory; the cluster on which our LS-DEM simulations were run has 48 gigabytes of RAM per processor, and the memory consumption of the entire simulation was around 1 gigabyte, which means that, only taking into account memory constraints, it would have been possible to run a simulation containing upwards of 140,000 grains. Furthermore, techniques to reduce the amount of memory used by the level set functions are possible, such as storing their values as floats instead of doubles and/or removing values either outside or very deep inside the particles by storing the level set functions in trees instead of on grids, but these techniques may affect accuracy or computational cost and remain areas to be researched.

\section{Conclusion}

Along with factors such as friction and void ratio, grain shape is an important factor that affects nearly every macroscopic quantity [5] of granular systems. We have presented a method, LS-DEM, to simulate systems of arbitrarily-shaped particles that can directly use outputs of level set-based characterization methods as its geometric basis. Furthermore, we have applied LS-DEM to two full virtual triaxial specimens with particle morphology coming straight from XRCT images of real experiments, being able to capture quantitatively, through LS-DEM, both the stress-strain and volume-strain relations observed in the experiments. By calibrating the parameters of our model to match the results of one experiment, we formulated a prediction of the behavior of the second experiment. Finally, we have highlighted some areas in which LS-DEM can be further explored, in mechanical, computational, and algorithmic respects.

\section{References}

[1] J. E. Andrade, C. F. Avila, N. Lenoir, S. A. Hall, and G. Viggiani. Multiscale modeling and characterization of granular matter: from grain scale kinematics to continuum mechanics. Journal of the Mechanics and Physics of Solids, 59:237-250, 2011.

[2] J. E. Andrade, K.-W. Lim, C. F. Avila, and I. Vlahinic. Granular element method for computational particle mechanics. Computer Methods in Applied Mechanics and Engineering, 241-244:262-274, 2012.

[3] J. Byerlee. Friction of rocks. Pure and Applied Geophysics, 116(4-5):615-626, 1978. 
[4] F. Casini, G. Viggiani, and S. Springman. Breakage of an artificial crushable material under loading. Granular Matter, 15(5):661-673, 2013.

[5] G. C. Cho, J. Dodds, and J. C. Santamarina. Particle shape effects on packing density, stiffness, and strength: Natural and crushed sands. Journal of Geotechnical and Geoenvironmental Engineering, 132(5):591-602, 2006.

[6] M. Cil and K. Alshibli. 3d assessment of fracture of sand particles using discrete element method. Géotechnique Letters, 2(July-September):161-166, 2012.

[7] M. Cil and K. Alshibli. 3d evolution of sand fracture under 1d compression. Géotechnique, 64(5):351-364, 2014.

[8] P. A. Cundall and O. D. L. Strack. A discrete numerical model for granular assemblies. Géotechnique, 29:47-65, 1979.

[9] C. Ericson. Real-Time Collision Detection (The Morgan Kaufmann Series in Interactive 3-D Technology) (The Morgan Kaufmann Series in Interactive 3D Technology). Morgan Kaufmann Publishers Inc., San Francisco, CA, USA, 2004.

[10] E. Hoskins, J. Jaeger, and K. Rosengren. A medium-scale direct friction experiment. International Journal of Rock Mechanics and Mining Sciences 86 Geomechanics Abstracts, 5(2):143 - 152, 1968.

[11] J. Jaeger. Failure of rocks under tensile conditions. International Journal of Rock Mechanics and Mining Sciences 65 Geomechanics Abstracts, 4(2):219-227, 1967.

[12] T. Laursen. Computational Contact and Impact Mechanics: Fundamentals of Modeling Interfacial Phenomena in Nonlinear Finite Element Analysis. Springer, Berlin, 2002.

[13] K. Lim, R. Kawamoto, E. Andò, G. Viggiani, and J. Andrade. Multiscale characterization and modeling of granular materials through a computational mechanics avatar: a case study with experiment. Acta Geotechnica, 2015.

[14] K.-W. Lim, K. Krabbenhoft, and J. Andrade. On the contact treatment of nonconvex particles in the granular element method. Computational Particle Mechanics, 1(3):257-275, 2014.

[15] S. Osher and R. Fedkiw. Level Set Methods and Dynamic Implicit Surfaces. Springer, 2003 edition edition, 2003.

[16] N. D. Parab, B. Claus, M. C. Hudspeth, J. T. Black, A. Mondal, J. Sun, K. Fezzaa, X. Xiao, S. Luo, and W. Chen. Experimental assessment of fracture of individual sand particles at different loading rates. International Journal of Impact Engineering, 68:8-14, 2014. 
[17] C. Sammis, G. King, and R. Biegel. The kinematics of gouge deformation. pure and applied geophysics, 125(5):777-812, 1987.

[18] K. Senetakis, M. R. Coop, and M. C. Todisco. The inter-particle coefficient of friction at the contacts of leighton buzzard sand quartz minerals. Soils and Foundations, 53(5):746 - 755, 2013.

[19] B. Shen, O. Stephansson, and M. Rinne. Modelling Rock Fracturing Processes. Springer Netherlands, 2014.

[20] X. Tu and J. E. Andrade. Criteria for static equilibrium in particulate mechanics computations. International Journal for Numerical Methods in Engineering, 75:1581-1606, 2008.

[21] I. Vlahinic, E. Ando, G. Viggiani, and J. E. Andrade. Towards a more accurate characterization of granular media: extracting quantitative descriptors from tomographic images. Granular Matter, pages 1-13, 2013. doi:10.1007/s10035-0130460-6.

[22] O. R. Walton and R. L. Braun, editors. Simulation of rotary-drum and repose tests for frictional spheres and rigid sphere clusters, Nov. 1993.

[23] W. Weibull. A statistical theory of strength of materials. In Proceedings of the Ingeniors Vetenskapsakad, 1939.

[24] W. Weibull. A statistical distribution of wide applicability. Journal of applied mechanics, 1951. 